\title{
A method superior to traditional spectral identification: FT-NIR two-dimensional correlation spectroscopy combined with deep learning to identify the shelf life of fresh Phlebopus portentosus
}

Li Wang, Jieqing Li, Tao Li, Honggao Liu *and Yuanzhong Wang *

\section{Author Information}

Corresponding Author

Honggao Liu - College of Agronomy and Life Sciences, Zhaotong University, Zhaotong 657000, China.

Email: honggaoliu@126.com

Yuanzhong Wang - Medicinal Plants Research Institute, Yunnan Academy of Agricultural Sciences, Kunming, 650200, China.

E-mail: boletus@126.com

Authors

Li Wang - College of Agronomy and Biotechnology, Yunnan Agricultural University, Kunming 650201, China. E-mail: wlwanglidong@126.com

Jieqing Li - College of Resources and Environment, Yunnan Agricultural University, Kunming 650201, China. E-mail: lijieqing2008@126.com

Tao Li -College of Resources and Environment, Yuxi Normal University, Yuxi 653199, China. E-mail: litao1976@yxnu.edu.cn 


\section{Figure Captions}

Figure. S1 The result and optimal hyperplane graph of SVM model prediction set based on different preprocessing methods. (1) RAW, (2) FD, (3) SD.

Figure. S2 PCA feature visualization of P. portentosus samples.

Figure. S3 The result graph of ELM model prediction set based on different preprocessing methods. (1) RAW, (2) FD, (3) SD.

\section{Table Captions}

Table S1. The confusion matrix of SVM, PLS-DA, ELM and DL model based on $P$. portentosus 

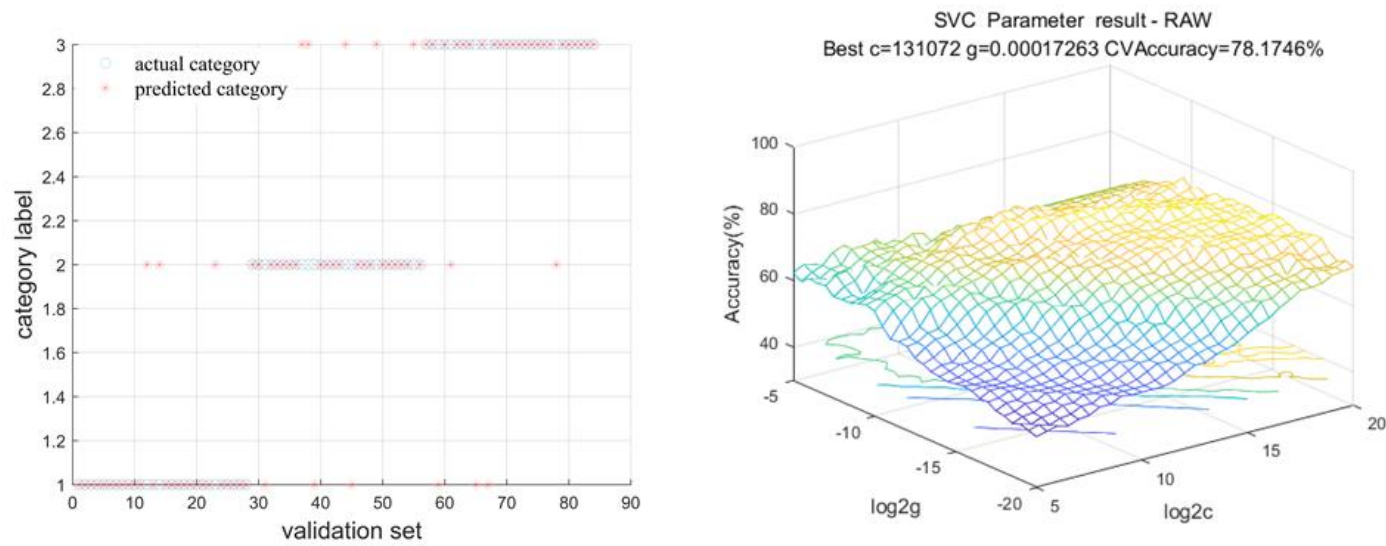

(1)
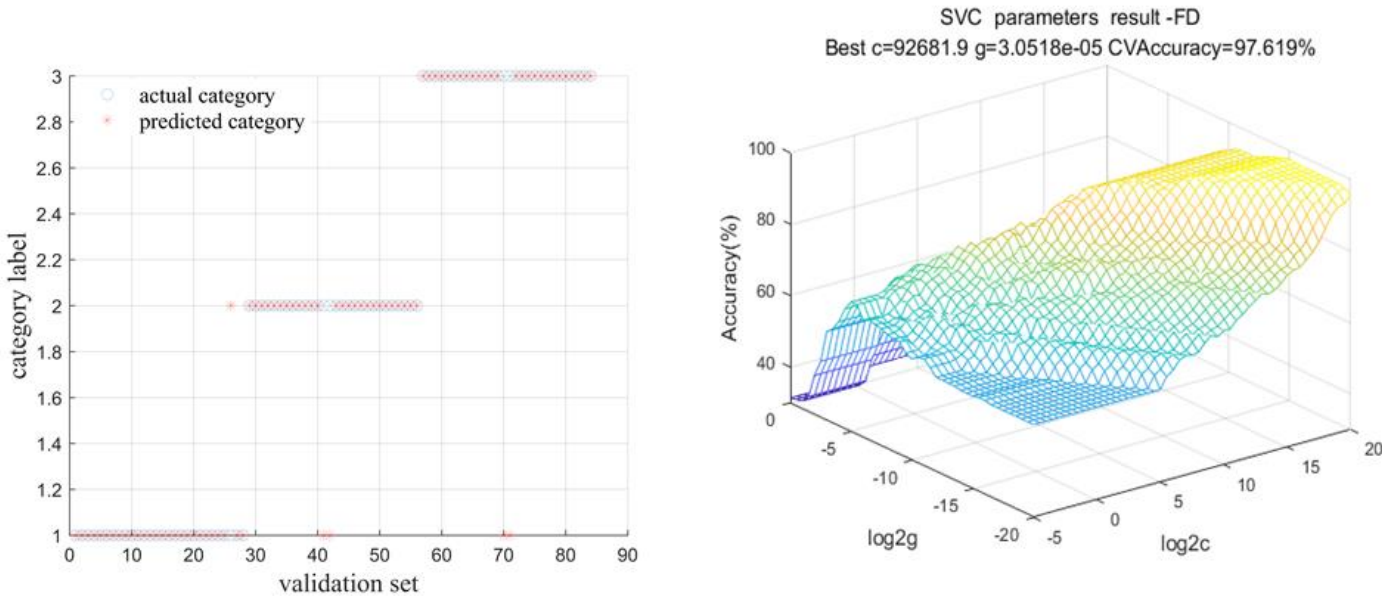

(2)
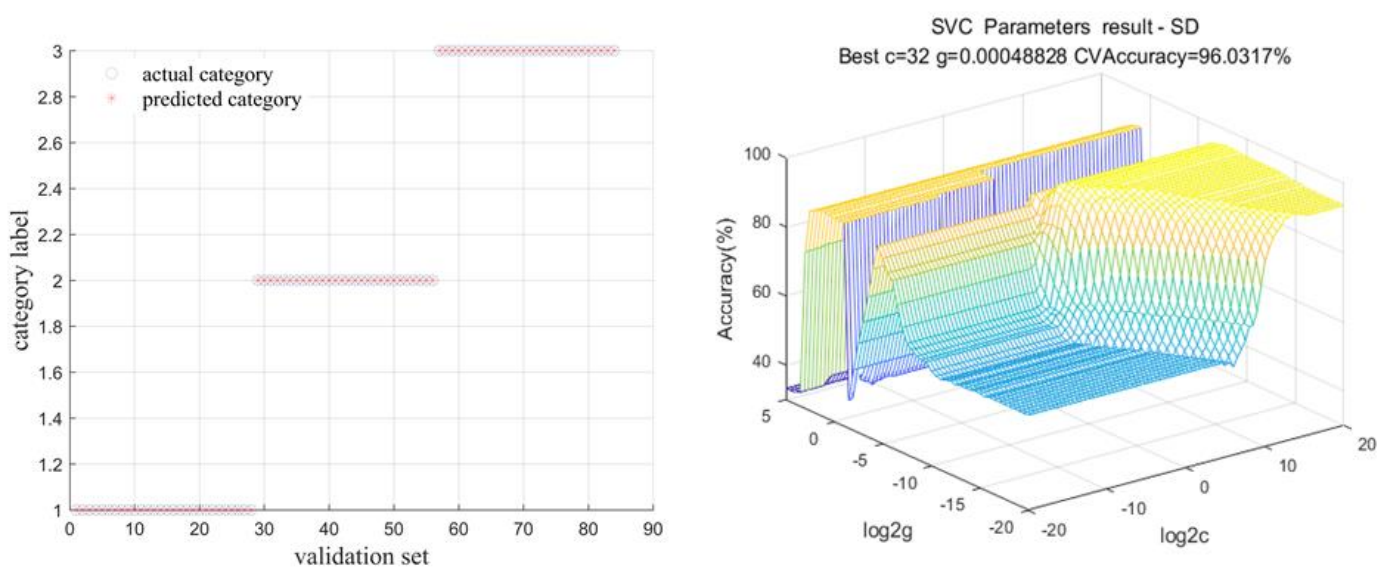

(3)

Figure. S1 The result graph of SVM model prediction set based on different preprocessing methods. (1) RAW, (2) FD, (3) SD. 
RAW.M1 (PCA-X)

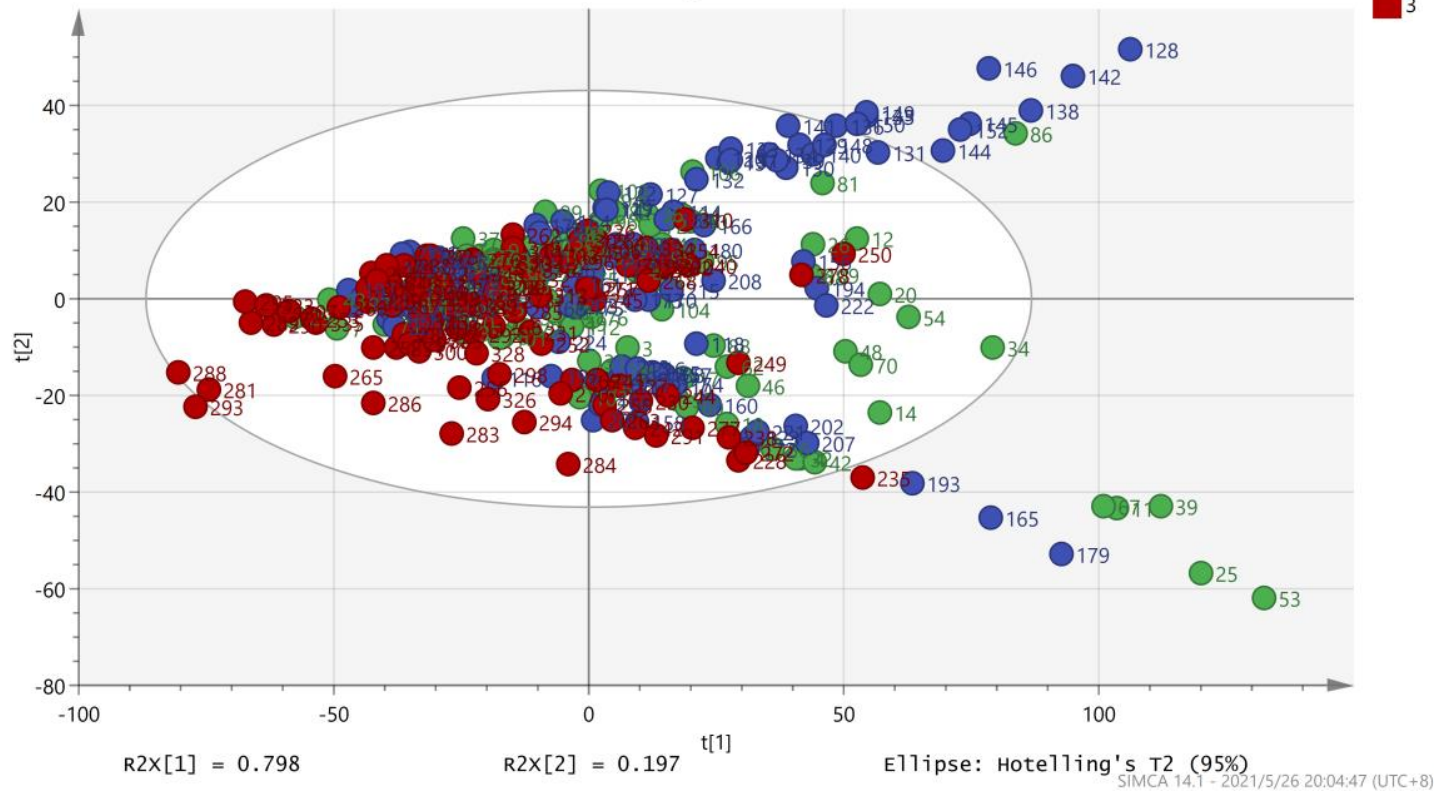

Figure. S2 PCA feature visualization of $P$. portentosus samples. 

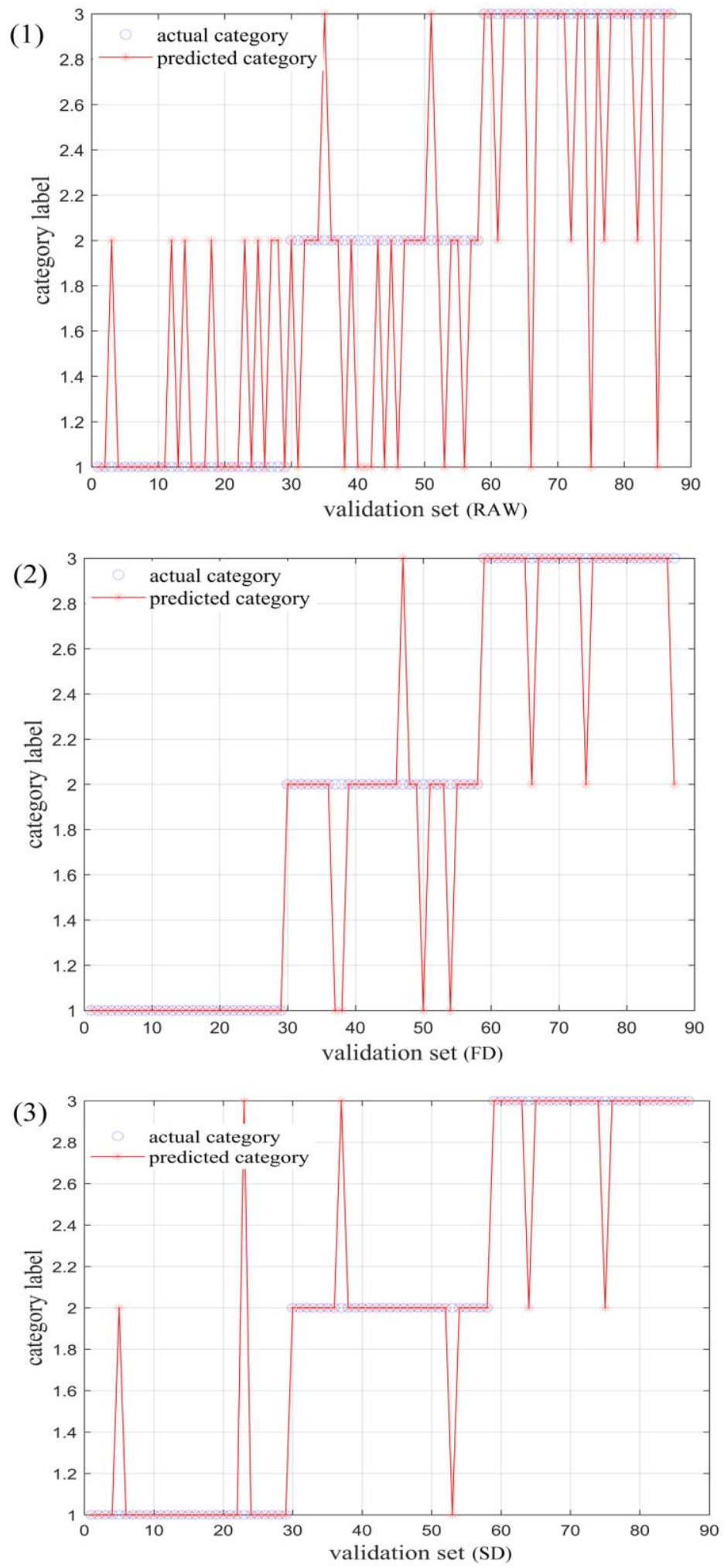

Figure. S3 The result graph of ELM model prediction set based on different preprocessing methods. (1) RAW, (2) FD, (3) SD. 
Table S1. The confusion matrix of SVM, PLS-DA, ELM and DL model based on $P$. portentosus

\begin{tabular}{|c|c|c|c|c|c|c|c|c|c|c|}
\hline \multirow{2}{*}{ Methods } & \multirow{2}{*}{ Predicted } & \multicolumn{3}{|c|}{ Raw } & \multicolumn{3}{|c|}{$\mathrm{FD}$} & \multicolumn{3}{|c|}{ SD } \\
\hline & & I & II & III & I & II & III & $\mathrm{I}$ & II & III \\
\hline \multirow[t]{3}{*}{ SVM } & I & 25 & 3 & 0 & 27 & 1 & 0 & 28 & 0 & 0 \\
\hline & II & 3 & 20 & 5 & 2 & 26 & 0 & 0 & 28 & 0 \\
\hline & III & 3 & 2 & 23 & 2 & 0 & 26 & 0 & 0 & 28 \\
\hline PLS- & I & 21 & 7 & 0 & 26 & 1 & 1 & 27 & 0 & 1 \\
\hline \multirow[t]{2}{*}{ DA } & II & 2 & 22 & 4 & 1 & 27 & 0 & 0 & 28 & 0 \\
\hline & III & 3 & 1 & 24 & 2 & 2 & 24 & 1 & 0 & 27 \\
\hline \multirow[t]{3}{*}{ ELM } & I & 21 & 8 & 0 & 29 & 0 & 0 & 27 & 1 & 1 \\
\hline & II & 7 & 20 & 2 & 4 & 24 & 1 & 1 & 27 & 1 \\
\hline & III & 3 & 4 & 22 & 0 & 3 & 26 & 0 & 2 & 27 \\
\hline \multirow[t]{3}{*}{ DL } & I & 10 & 0 & 0 & 1 & 1 & 1 & 1 & 1 & 1 \\
\hline & II & 0 & 10 & 0 & 1 & l & I & l & l & 1 \\
\hline & III & 0 & 0 & 10 & 1 & 1 & 1 & 1 & 1 & 1 \\
\hline
\end{tabular}

